\title{
Uso do indutor de resistência associado a diferentes fungicidas na cultura da soja
}

\author{
Use of resistance inducer associated with different fungicides in soybean \\ Uso de inductor de resistencia asociado a diferentes fungicidas en soja
}

Recebido: 28/12/2021 | Revisado: 03/01/2022 | Aceito: 11/01/2022| Publicado: 13/01/2022

Wendson Soares da Silva Cavalcante

ORCID: https://orcid.org/0000-0002-5224-5486 Universidade de Rio Verde, Brasil E-mail: wendsonbfsoarescvt@gmail.com

Nelmício Furtado da Silva

ORCID: https://orcid.org/0000-0001-7055-8075

Universidade de Rio Verde, Brasil

E-mail: nelmiciofurtado@gmail.com

Giacomo Zanotto Neto

ORCID: https://orcid.org/0000-0001-8167-5066 Instituto Federal de Educação, Ciência e Tecnologia Goiano, Brasil E-mail: giacomozn@gmail.com

Marconi Batista Teixeira

ORCID: https://orcid.org/0000-0002-0152-256X Instituto Federal de Educação, Ciência e Tecnologia Goiano, Brasil E-mail: marconi.teixeira@ifgoiano.edu.br

Fernando Rodrigues Cabral Filho

ORCID: https://orcid.org/0000-0002-5090-5946 Instituto Federal de Educação, Ciência e Tecnologia Goiano, Brasil E-mail: fernandorcfilho@hotmail.com

Fernando Nobre Cunha

ORCID: https://orcid.org/0000-0001-8489-7625 Instituto Federal de Educação, Ciência e Tecnologia Goiano, Brasil

E-mail: fernandonobrecunha@ hotmail.com

Fernando Rezende Corrêa

ORCID: https://orcid.org/0000-0001-7110-3611 Faculdade Rio Verde, Brasil E-mail: fernandorvcorrea@gmail.com

Guilherme Rodrigues Martins

ORCID: https://orcid.org/0000-0002-1014-1232 Universidade de Rio Verde, Brasil

E-mail: guilhermemartinsrv5@gmail.com

\begin{abstract}
Resumo
As fitoalexinas constituem um grupo de metabólitos secundários quimicamente diversos, antimicrobianos, de baixa massa molecular, com forte atividade antimicrobiana e que se acumulam em torno do local de infecção. Partindo da hipótese que o uso de indutores de resistência associado a aplicação de fungicida promove um maior controle de doenças na cultura da soja, objetivou-se no presente estudo quantificar a incidência e severidade e doenças em função do uso de indutor de resistência associado a aplicação de fungicida na cultura da soja. O experimento foi desenvolvido na área de experimentação pertencente a empresa Tecno Nutrição Vegetal e Biotecnologia Ltda. O delineamento experimental utilizado foi de blocos casualizados com quatro repetições. Os tratamentos consistiram na aplicação de 5 combinações de bioestimulantes: T1) Fox (45; 65; e 85 DAP); T2) Fox + Fitalexy (45 DAP), Fox (65 e 85 DAP); T3) Fox + Fitalexy (45 e 65 DAP) e Fox (85 DAP); T4) Priori Xtra (45, 65 e 85 DAP); T5) Priori Xtra + Fitalexy (45 DAP) e Priori Xtra (65 e 85 DAP); T6) Priori Xtra + Fitalexy (45 e 65 DAP) e Priori Xtra (85 DAP); T7) Aproach Prima (45, 65 e 85 DAP); T8) Aproach Prima + Fitalexy (45 DAP) e Aproach Prima (65 e 85 DAP); T9) Aproach Prima + Fitalexy (45 e 65 DAP) e Controle. Foram mensuradas as variáveis severidade de doenças e de produtividade dos grãos da soja. Os dados foram submetidos a análise de variância $(p<0,05)$ e ao teste de média Tukey $(p<0,05)$. O indutor de resistência em combinação com fungicidas reduziram a severidade e a incidência de doenças com um ou duas aplicações, bem como promoveram incrementos significativos na produtividade de grãos de soja.
\end{abstract}

Palavras-chave: Fitalexy ${ }^{\circledR}$; Fitoalexinas; Severidade; Doenças; Produtividade.

\section{Abstract}

Phytoalexins are a group of chemically diverse, antimicrobial, low molecular weight secondary metabolites with strong antimicrobial activity that accumulate around the site of infection. Based on the hypothesis that the use of resistance inducers associated with fungicide application promotes greater control of diseases in soybean, the objective of this study was to quantify the incidence and severity of diseases as a function of the use of resistance 
inducers associated with application. of fungicide in the soybean crop. The experiment was developed in the experimental area belonging to the company Tecno Nutrição Vegetal e Biotecnologia Ltda. The experimental design used was randomized blocks with four replications. The treatments consisted of the application of 5 combinations of biostimulants: T1) Fox (45; 65; and 85 DAP); T2) Fox + Fitalexy (45 DAP), Fox (65 and 85 DAP); T3) Fox + Fitalexy (45 and 65 DAP) and Fox (85 DAP); T4) Priori Xtra (45, 65 and 85 DAP); T5) Priori Xtra + Fitalexy (45 DAP) and Priori Xtra (65 and 85 DAP); T6) Priori Xtra + Fitalexy (45 and 65 DAP) and Priori Xtra (85 DAP); T7) Approach Prima (45, 65 and 85 DAP); T8) Approach Prima + Fitalexy (45 DAP) and Aproach Prima (65 and 85 DAP); T9) Prima Approach + Fitalexy (45 and 65 DAP) and Control. The variables severity of diseases and soybean grain yield were measured. Data were submitted to analysis of variance $(\mathrm{p}<0.05)$ and the Tukey mean test $(\mathrm{p}<0.05)$. The resistance inducer in combination with fungicides reduced the severity and incidence of diseases with one or two applications, as well as promoting significant increases in soybean yield.

Keywords: Fitalexy ${ }^{\circledR}$; Phytoalexins; Severity; Illnesses; Productivity.

\section{Resumen}

Las fitoalexinas son un grupo de metabolitos secundarios químicamente diversos, antimicrobianos y de bajo peso molecular con fuerte actividad antimicrobiana que se acumulan alrededor del sitio de la infección. Partiendo de la hipótesis de que el uso de inductores de resistencia asociados a la aplicación de fungicidas promueve un mayor control de enfermedades en soja, el objetivo de este estudio fue cuantificar la incidencia y severidad de las enfermedades en función del uso de inductores de resistencia asociados a la aplicación de fungicida en el cultivo de soja. El experimento se desarrolló en el área experimental perteneciente a la empresa Tecno Nutrição Vegetal e Biotecnologia Ltda. El diseño experimental utilizado fue bloques al azar con cuatro repeticiones. Los tratamientos consistieron en la aplicación de 5 combinaciones de bioestimulantes: T1) Fox (45; 65; y 85 DAP); T2) Fox + Fitalexy (45 DAP), Fox (65 y 85 DAP); T3) Fox + Fitalexy (45 y 65 DDP) y Fox (85 DDP); T4) Priori Xtra (45, 65 y 85 DAP); T5) Priori Xtra + Fitalexy (45 DDP) y Priori Xtra (65 y 85 DDP); T6) Priori Xtra + Fitalexy (45 y 65 DDP) y Priori Xtra (85 DDP); T7) Aproximación Prima (45, 65 y 85 DAP); T8) Abordaje Prima + Fitalexy (45 DAP) y Abordaje Prima (65 y 85 DAP); T9) Abordaje Prima + Fitalexy (45 y 65 DAP) y Control. Se midieron las variables severidad de enfermedades y rendimiento de grano de soja. Los datos fueron sometidos a análisis de varianza $(p<0,05)$ y prueba de medias de Tukey $(\mathrm{p}<0,05)$. El inductor de resistencia en combinación con fungicidas redujo la severidad e incidencia de enfermedades con una o dos aplicaciones, además de promover aumentos significativos en el rendimiento de la soja.

Palabras clave: Fitalexy ${ }^{\circledR}$; Fitoalexinas; Gravedad; Enfermedades; Productividad.

\section{Introdução}

O Brasil é mundialmente o maior produtor de soja (Glycine max (L.) Merrill), com uma produção de 124,2 milhões de toneladas; 36,820 milhões de hectares plantados com uma produtividade de $3.373 \mathrm{~kg} \mathrm{ha}^{-1}$, safra 2019/20 (CONAB, 2020). A produtividade da soja é influenciada por diversos fatores bióticos e abióticos, o que torna o manejo e tratos culturais ferramentas indispensáveis para o sucesso da lavoura.

$\mathrm{O}$ ataque de fitopatógenos e sua disseminação quando expostos a condições ideais para o seu desenvolvimento é muito rápido, o que reflete na diminuição do potencial genético e produtivo, e consequentemente na queda de produtividade (Ribeiro et al., 2017). As doenças foliares acarretam perdas que variam entre 15 a $20 \%$ da produção, mas algumas doenças podem ocasionar perdas de quase 100\% (Juhász et al., 2013). Um dos principais desafios para o cultivo da soja é o manejo fitossanitário de doenças (Fonseca \& Araújo, 2015).

Entre as alternativas de manejo de doenças que podem reduzir a severidade e as perdas decorrentes de doenças está o controle químico. A aplicação de duas ou mais técnicas, de manejo de doenças, integradas com o correto entendimento de doses e intervalo de aplicação de produtos, é um caminho para um cultivo de altas produtividades e qualidade. Os fungicidas além de serem aplicados de forma isolada, têm sido comumente associados a outros compostos, o que tem apresentado resultados satisfatórios, aumentando a eficácia no manejo de doenças das mais variadas culturas, principalmente no que tange o manejo da cultura da soja, a combinação de fungicida com substâncias que apresentam o efeito aditivo e sinérgico, quando utilizados em associação (Meneghetti et al., 2010; Ioris Junior, 2019).

Afim de reduzir a resistência de patógenos aos ingredientes ativos dos fungicidas, haja em vista a possibilidade de vulnerabilidade (Ribeiro et al., 2019), devem ser empregas outras estratégias no manejo de doenças. Tem-se buscado fazer 
novas associações e o uso de novas metodologias, como o uso do controle alternativo, e na busca de mecanismos que posam reduzir os impactos ambientais e na saúde, dentre eles pode-se citar a indução de resistência (Jacinto, 2019).

A indução a resistência é descrita na literatura como uma defesa estimulada em caráter sistêmico, a qual envolve a ativação de mecanismos de defesa existentes nas plantas, seja por fatores bióticos ou fatores abióticos no controle eficaz contra patógenos sejam eles bactérias, vírus, nematoides e fungos. A indução de resistência envolve a ativação de mecanismos de resistência nas plantas em resposta ao ataque de patógenos (Carvalho, 2012; Bertoldo \& Mazaro, 2018).

$\mathrm{O}$ fosfito $\left(\mathrm{H}_{2} \mathrm{PO}_{3}{ }^{-}\right.$e $\left.\mathrm{HPO}_{3}{ }^{-2}\right)$ são compostos oriundos da neutralização do ácido fosforoso $\left(\mathrm{H}_{3} \mathrm{PO}_{3}\right)$, que possuem uma alta atividade fungicida, estimulando as plantas a uma possível ativação de mecanismos de defesa, como a produção de metabolitos secundários, que conferem a planta uma maior produção de fitoalexinas (Guest \& Grant, 1991; Lovatt \& Mikkelsen, 2006).

As fitoalexinas constituem um grupo de metabólitos secundários quimicamente diversos, antimicrobianos, de baixa massa molecular, com forte atividade antimicrobiana e que se acumulam em torno do local de infecção (Taiz \& Zeiger, 2017).

Partindo da hipótese que o uso de indutores de resistência associado a aplicação de fungicida promove um maior controle de doenças na cultura da soja, objetivou-se no presente estudo quantificar a incidência e severidade e doenças em função do uso de indutor de resistência associado a aplicação de fungicida na cultura da soja.

\section{Metodologia}

O experimento foi desenvolvido na área de experimentação pertencente a empresa Tecno Nutrição Vegetal e Biotecnologia Ltda, na seguinte localização geográfica $17^{\circ} 44^{\prime} 20.88^{\prime}$ 's e $50^{\circ} 57^{\prime} 55.79$ '’O, com 860 m de altitude. O solo da área experimental é classificado como Latossolo Vermelho distróferrico (LVdf) (SANTOS et al., 2018), cuja características químicas e granulométricas estão descritas na Tabela 1.

Tabela 1. Análise química e granulométrica do solo, safra 2018-19, Rio Verde - GO.

\begin{tabular}{|c|c|c|c|c|c|c|c|c|c|c|c|c|c|}
\hline \multicolumn{14}{|c|}{ Macronutrientes } \\
\hline Prof. & $\mathrm{pH}$ & $\mathrm{P}$ & $\mathrm{S}$ & $\mathrm{K}$ & $\mathrm{Ca}$ & $\mathrm{Mg}$ & $\mathrm{Al}$ & $\mathrm{H}+\mathrm{Al}$ & M.O. & SB & CTC & $\mathrm{V}$ & $\mathrm{m}$ \\
\hline $\mathrm{cm}$ & $\mathrm{CaCl}_{2}$ & \multicolumn{3}{|c|}{$\mathrm{mg} \mathrm{dm}^{-3}$} & \multicolumn{3}{|c|}{........ $\mathrm{cmol}_{\mathrm{c}} \mathrm{dm}^{-3}$} & & $\mathrm{~g} \mathrm{dm}^{-3}$ & \multicolumn{2}{|c|}{$\mathrm{cmol}_{\mathrm{c}} \mathrm{dm}^{-3}$} & \multicolumn{2}{|c|}{$\%$} \\
\hline $0-20$ & 5,18 & 1,83 & 3,15 & 12,81 & 1,6 & 1,22 & 0,00 & 3,07 & 37,33 & 3,15 & 6,31 & 49,8 & 49,6 \\
\hline $20-40$ & 5,23 & 0,83 & 2,53 & 4,03 & 1,2 & 0,83 & 0,00 & 2,5 & 24,0 & 2,17 & 4,17 & 47,6 & 52,3 \\
\hline \multicolumn{8}{|c|}{ Micronutrientes } & \multicolumn{6}{|c|}{ Granulometria } \\
\hline & B & $\mathrm{Na}$ & $\mathrm{Cu}$ & $\mathrm{Fe}$ & $\mathrm{Mn}$ & $\mathrm{Zn}$ & & Areia & Silte & Argila & \multicolumn{3}{|c|}{ Classe textural } \\
\hline & & ........... & $\ldots . . \mathrm{mg}$ & $-3 \ldots \ldots$ & & & & & $\%$ & & \\
\hline $0-20$ & 0,11 & 0,0 & 1,77 & 16,9 & 11,3 & 1,8 & & 23,4 & 11,5 & 65,0 & \multicolumn{3}{|c|}{ M. Argiloso } \\
\hline $20-40$ & 0,0 & 0,0 & 0,0 & 0,0 & 0,0 & 1,5 & & 19,6 & 12,0 & 68,3 & \multicolumn{3}{|c|}{ M. Argiloso } \\
\hline
\end{tabular}

pH da solução do solo, determinado em solução de cloreto de cálcio; MO: matéria orgânica, determinação por método colorimétrico; P: fósforo, melhich; $\mathrm{K}^{+}$: potássio, melhich; $\mathrm{Ca}^{2+} \mathrm{e} \mathrm{Mg}^{2+}$ : teores trocáveis de cálcio e magnésio, respectivamente, em $\mathrm{KCl}_{3} \mathrm{~S}_{-} \mathrm{SO}^{4}{ }_{2-:}$ Enxofre na forma de sulfatos, extraído por fosfato de cálcio e determinado por colorimetria. $\mathrm{Al}^{3+}$ : Alumínio trocável, extraído por solução de cloreto de potássio a $1 \mathrm{~mol} \mathrm{~L}^{-1}$. $\mathrm{H}+\mathrm{Al}$ : acidez total do solo, determinada em solução tampão $\mathrm{SMP}$ a pH 7,5. SB: soma de bases $\left(\mathrm{K}^{+}+\mathrm{Ca}^{2+}+\mathrm{Mg}^{2+}\right)$. CTC: capacidade de troca de cátions $\left(\mathrm{K}++\mathrm{Ca}^{2+}+\mathrm{Mg}^{2+}+\mathrm{H}+\mathrm{Al}\right)$. V: saturação por bases do solo (relação SB/CTC). m: saturação por alumínio [relação $\left.\mathrm{Al}^{3+} /\left(\mathrm{SB}+\mathrm{Al}^{3+}\right)\right]$. $\mathrm{Cu}, \mathrm{Fe}, \mathrm{Mn}$ e $\mathrm{Zn}$ : cobre, ferro, manganês e zinco, extraídos por solução melhich.

Segundo a classificação de Alvares et al. (2013) e Köppen e Geiger (1928), clima da região é classificado como Aw (tropical), com chuva nos meses de outubro a maio, e com seca nos meses de junho a setembro. A temperatura média anual varia de 20 a $35{ }^{\circ} \mathrm{C}$ e as precipitações variam de 1.500 a $1.800 \mathrm{~mm}$ anuais e o relevo é suave ondulado (6\% de declividade). Durante o desenvolvimento da cultura os dados climáticos locais, foram monitorados, e as médias semanais dispostas na Figura 1. 
Figura 1. Dados diários, precipitação, temperatura e umidade relativa no período decorrente do experimento, Rio Verde - GO, $2018 / 19$.

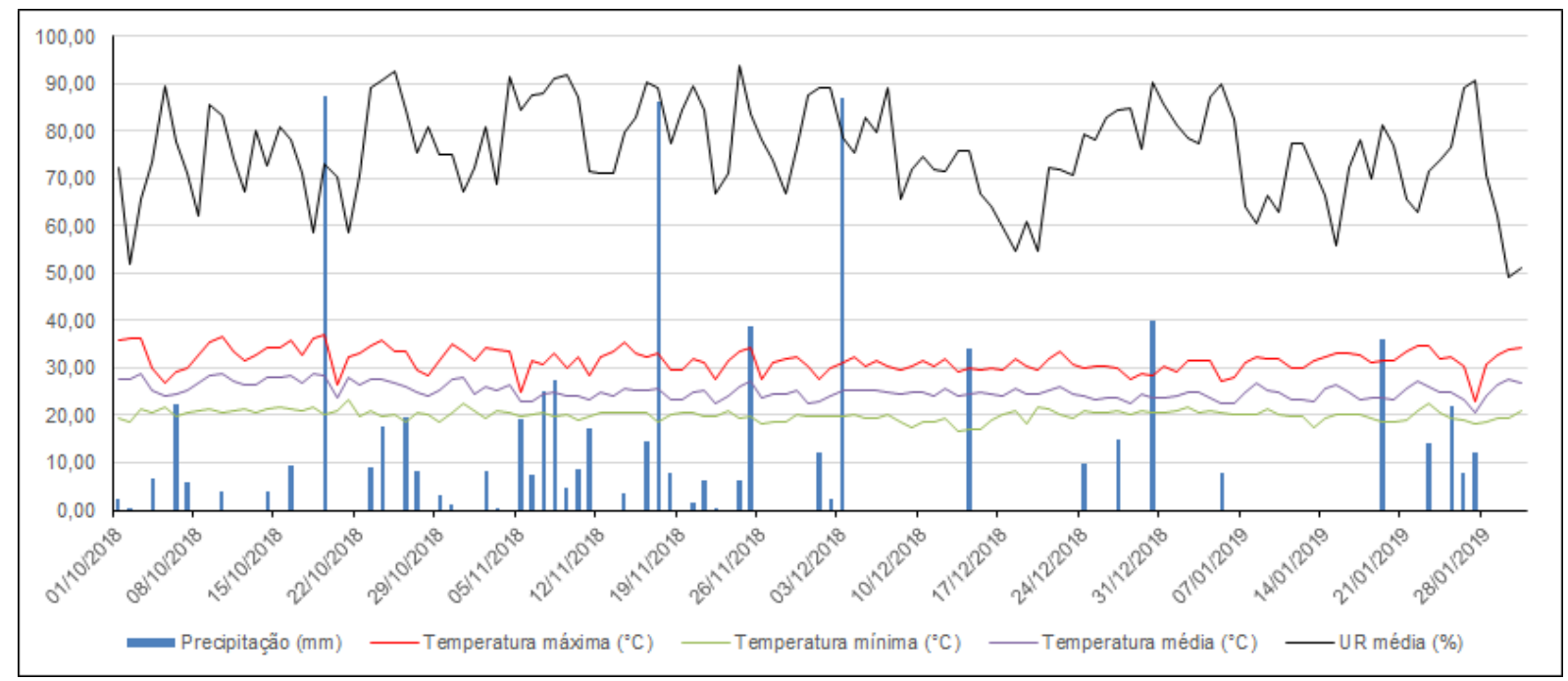

Fonte: Estação Normal INMET - Safrinha 2018-19, Rio Verde - GO.

A variedade utilizada foi a Brasmax Bônus 8579RSF IPRO, sendo depositadas 14 sementes por metro, totalizando uma população de plantas de 280 mil plantas ha-1, a variedade mais utilizada na região. O plantio da soja foi realizado dia 10/11/2018 no sistema de plantio direto, utilizando uma semeadora Jumil de 10 linhas, mecânica, equipada com mecanismo de abertura de sulco do tipo disco duplo.

Durante o desenvolvimento da cultura foram feitos os tratos culturais via aplicações de produtos químicos para o controle de plantas daninhas, pragas e doenças, conforme Tabela 2.

Tabela 2. Número de aplicações, época, dose, produto comercial e princípios ativos utilizados durante o cultivo da soja, safra 2018-19, Rio Verde - GO.

\begin{tabular}{|c|c|c|}
\hline Aplicação & Época & Dose e produto comercial e princípios ativos \\
\hline $1^{\text {a }}$ Dessecação & Pré-plantio & $\begin{array}{c}\text { 3,0 } \mathrm{L} \mathrm{ha}^{-1} \text { de Crucial (Glifosato) }+0,5 \mathrm{~L} \mathrm{ha}^{-1} \text { de Zethamaxx (Flumioxazina + Imazetapir) } \\
+0,6 \mathrm{~L} \mathrm{ha}^{-1} \mathrm{de} 2,4-\mathrm{D}(\text { Dez). }\end{array}$ \\
\hline TS & Semeadura & $\begin{array}{l}0,5 \mathrm{~L} / 100 \mathrm{~kg} \text { de semente de Cropstar (Tiodicarbe + Imidacloprido) + Protreat (Tiram + } \\
\text { Carbendazin) }+0,1 \mathrm{~L} / 100 \mathrm{~kg} \text { de semente de Nodumax (Bradyrhizobium japonicum })+0,1 \\
\mathrm{~L} / 100 \mathrm{~kg} \text { de semente de Nodumax gramínea (Azospirillum brasilense). }\end{array}$ \\
\hline Pós-emergente & 20DAE & 2,0 L ha ${ }^{-1}$ de Crucial (Glifosato) $+0,8 \mathrm{~L} \mathrm{ha}^{-1}$ de Cletodim (Viance). \\
\hline $1^{a}$ Inseticida & $30 \mathrm{DAE}$ & $0,07 \mathrm{~L} \mathrm{ha}^{-1}$ de Kaiso (Lambda-cialotrina). \\
\hline $2^{\mathrm{a}}$ Inseticida & $45 \mathrm{DAE}$ & 0,07 L ha ${ }^{-1}$ de Kaiso (Lambda-cialotrina) $+0,2 \mathrm{~L} \mathrm{ha}^{-1}$ de Valio (Óleo de laranja). \\
\hline $3^{\mathrm{a}}$ Inseticida & $60 \mathrm{DAE}$ & $1,0 \mathrm{~kg} \mathrm{ha}^{-1}$ de Perito (Acefato) $+0,2 \mathrm{~L} \mathrm{ha}^{-1}$ de Valio (Óleo de laranja). \\
\hline $4^{\mathrm{a}}$ Inseticida & 75 DAE & $\begin{array}{c}0,07 \mathrm{~L} \mathrm{ha}^{-1} \text { de Kaiso (Lambda-cialotrina) }+1,0 \mathrm{~kg} \mathrm{ha}^{-1} \text { de Perito (Acefato) }+0,2 \mathrm{~L} \mathrm{ha}^{-1} \mathrm{de} \\
\text { Valio (Óleo de laranja). }\end{array}$ \\
\hline Dessecação & $110 \mathrm{DAE}$ & 2,0 L ha ${ }^{-1}$ de Paraquat (Helmoxone) $+0,4 \mathrm{~L} \mathrm{ha}^{-1}$ de Valio (Óleo de laranja). \\
\hline
\end{tabular}

DAE - dias após a emergência. Fonte: Autores.

O delineamento experimental utilizado foi o de blocos casualizados com quatro repetições, sendo que as parcelas experimentais continham 8 fileiras espaçadas em $0,45 \mathrm{~m}$ e $10 \mathrm{~m}$ de comprimento com bordaduras de $2 \mathrm{~m}$ entre parcelas e 0,90 $\mathrm{m}$ entre blocos.

Os tratamentos, formulações, dose e estádio de aplicação são descritos conforme Tabela 3. As aplicações dos tratamentos foram feitas conforme o manejo fitossanitário realizado em áreas comerciais e ainda os momentos ideias para o controle de doenças. 
Research, Society and Development, v. 11, n. 1, e51711125204, 2022

(CC BY 4.0) | ISSN 2525-3409 | DOI: http://dx.doi.org/10.33448/rsd-v11i1.25204

Tabela 3. Descrição dos diferentes tratamentos, safra 2018-19, Rio Verde - GO.

\begin{tabular}{|c|c|c|c|}
\hline \multirow{3}{*}{$\mathrm{N}^{\circ}$} & \multicolumn{3}{|c|}{ Tratamentos } \\
\hline & $1^{a}$ Fungicida & $2^{\mathrm{a}}$ Fungicida & $3^{\mathrm{a}}$ Fungicida \\
\hline & 45 DAP & 65 DAP & 85 DAP \\
\hline 1 & Fox $^{1}$ & Fox & Fox \\
\hline 2 & Fox + Fitalexy $^{6}$ & Fox & Fox \\
\hline 3 & Fox + Fitalexy & Fox + Fitalexy & Fox \\
\hline 4 & Priori Xtra ${ }^{2}$ & Priori Xtra & Priori Xtra \\
\hline 5 & Priori Xtra + Fitalexy & Priori Xtra & Priori Xtra \\
\hline 6 & Priori Xtra + Fitalexy & Priori Xtra + Fitalexy & Priori Xtra \\
\hline 7 & Aproach Prima $^{3}$ & Aproach Prima & Aproach Prima \\
\hline 8 & Aproach Prima + Fitalexy & Aproach Prima & Aproach Prima \\
\hline 9 & Aproach Prima + Fitalexy & Aproach Prima + Fitalexy & Aproach Prima \\
\hline Controle & - & - & - \\
\hline
\end{tabular}

${ }^{1} 0,4 \mathrm{~L} \mathrm{ha}^{-1}$ de Fox ${ }^{1}$ (Protioconazol + Trifloxistrobina) + 0,25\% de Aureo; ${ }^{2} 0,3 \mathrm{~L} \mathrm{ha}^{-1}$ de Priori Xtra (Azoxistrobina + Ciproconazol) + 0,5\% de Nimbus; ${ }^{3}$ 0,3 $\mathrm{L} \mathrm{ha}^{-1}$ de Aproach Prima (Picoxistrobina + Ciproconazol) + 0,5\% de Óleo; ${ }^{4} 0,2 \mathrm{~kg} \mathrm{ha}^{-1},{ }^{6} 0,5 \mathrm{~L} \mathrm{ha}^{-1}$ de Fitalexy (Fosfito de $\mathrm{Cu})$. Fonte: Autores.

As aplicações dos tratamentos foram feitas utilizando um pulverizador costal com pressurização por $\mathrm{CO}_{2}$ munido de barra de $2 \mathrm{~m}$, contendo quatro pontas de pulverização do tipo TT 110.02 (0,45 m entre pontas), aplicando volume de calda equivalente a $100 \mathrm{~L} \mathrm{ha}^{-1}$. As condições ambientais foram sempre monitoradas para obter uma condição favorável de temperatura média $25^{\circ} \mathrm{C}$, UR média de $78 \%$ e velocidade do vento média de $2,5 \mathrm{~km} \mathrm{~h}^{-1}$. As aplicações foram sempre realizadas entre 8:00 e 10:00 horas ou das 16 às 18 horas, período que foi possível reunir as melhores condições climáticas para as aplicações.

Foram realizadas avaliações visuais de doenças foliares (100 dias após o plantio - DAP) com o objetivo de avaliar a sanidade das plantas. Foi determinado as variáveis severidade (\%) de doenças foliares.

Foram avaliadas as plantas contidas em $1 \mathrm{~m}$ de linha no centro das parcelas nas 4 repetições, totalizando $4 \mathrm{~m}$ de linhas por tratamento, localizadas aleatoriamente nas linhas destinadas para avaliações destrutivas. A incidência foi determinada conforme a Equação 1.

\section{Incidência $(\%)=n^{0}$ de plantas com sintomas $x$ 100/n $\mathbf{n}^{\circ}$ total de plantas (Eq. 1)}

A severidade foi determinada pela escala diagramática das doenças de final de ciclo (DFC) da soja (Glycine max) (Martins et al., 2004) (Figura 2). 
Figura 2. Escala diagramática das doenças de final de ciclo da soja (Glycine max). Painel superior: Sintomas agregados. Painel inferior: Sintomas aleatoriamente distribuídos (Martins et al., 2004).
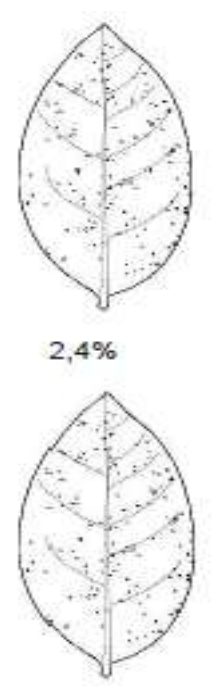

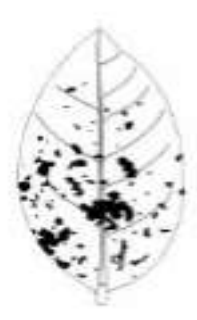

$15,2 \%$

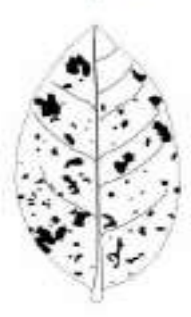

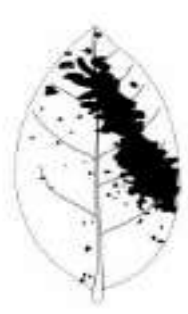

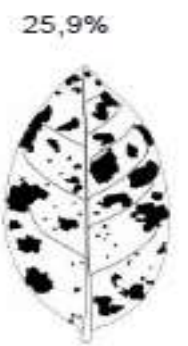

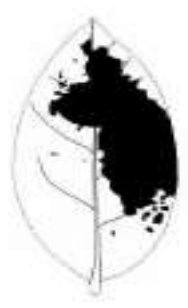

$40,5 \%$

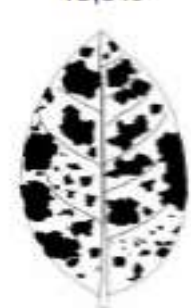

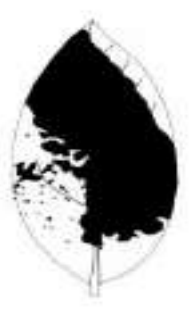

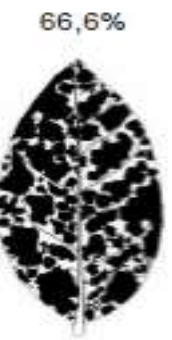

Fonte: Autores.

No final do ciclo foi determinada a massa de 100 grãos (M100G em g) a produtividade de grãos (PG em kg ha $\left.{ }^{-1}\right)$. Foram colhidas $2 \mathrm{~m}$ das 2 linhas centrais $\left(2 \mathrm{~m} \mathrm{x} 1 \mathrm{~m}=2 \mathrm{~m}^{2}\right.$ por parcela, totalizando $8 \mathrm{~m}^{2}$ por tratamento.

Os dados foram submetidos a análise de variância $(p<0,05)$ e os casos de significância foram submetidos ao teste de média Tukey $(p<0,05)$, utilizando o software estatístico SISVAR ${ }^{\circledR}$ (Ferreira, 2011).

\section{Resultados e Discussão}

As doenças de final de ciclo (DFC) causadas pela presença de patógenos como Septoria glycines (mancha parda), Cercospora kikuchii (crestamento foliar por cercospora e mancha púrpura), Cercospora sojina (mancha olho de rã) e Colletotrichum truncatum (antracnose) ocorreram por infestação natural no campo, sem inoculação artificial.

As análises demostraram que houve diferenças significativas para as variáveis analisadas (Tabela 4).

Tabela 4. Resumo da análise de variância para a variável severidade de doenças (SD \%), em função dos tratamentos, safra 2018-19, Rio Verde - GO.

\begin{tabular}{ccc}
\hline FV & \multirow{2}{*}{ GL } & Quadrados médios (QM) \\
\cline { 3 - 3 } Tratamentos & 9 & SD \% \\
Blocos & 3 & $115,1367^{* *}$ \\
Resíduos & 27 & 3,5816 \\
CV $(\%)$ & & 12,20 \\
\hline
\end{tabular}

${ }^{\text {ns }}$ não significativo e $*$; ** significativo respectivamente a 5 e $1 \%$ de probabilidade segundo teste F. GL - Grau de Liberdade e CV Coeficiente de Variação. Fonte: Autores.

$\mathrm{Na}$ avaliação da severidade de doenças (SD \%) o uso do indutor de resistência associado a aplicação de diferentes fungicidas promoveram diminuição na incidência das DFC corroborando em um maior controle de doenças (Tabela 4). O tratamento controle, ou seja, onde não foram feitas aplicações de fungicida e nem a combinação com indutor resistência, obteve o percentual de severidade de $20,66 \%$ o maior nível de incidência e severidade de doenças (Tabela 5). 
Tabela 5. Teste de média para a severidade (\%) de doenças de final de ciclo nos diferentes tratamentos, safra 2018-19, Rio Verde - GO

\begin{tabular}{|c|c|}
\hline \multirow{2}{*}{ Tratamentos } & Médias \\
\hline & SD \% \\
\hline \multicolumn{2}{|r|}{ Ad } \\
\hline 1 & $7,10 \mathrm{~d}$ \\
\hline 2 & $7,10 \mathrm{~d}$ \\
\hline 3 & $6,29 d$ \\
\hline 4 & $19,87 \mathrm{~b}$ \\
\hline 5 & $14,54 \mathrm{c}$ \\
\hline 6 & $19,87 \mathrm{c}$ \\
\hline 7 & $26,64 \mathrm{a}$ \\
\hline 8 & $19,87 \mathrm{~b}$ \\
\hline 9 & $7,10 \mathrm{~d}$ \\
\hline Controle & 26,70 \\
\hline Erro padrão & 0,9462 \\
\hline
\end{tabular}

Ad - adimensional; Valores médios da severidade $(\%)$ de doenças de final de ciclo. Fonte: Autores.

Todos os tratamentos com uso isolado dos fungicidas ou em combinações dos fungicidas com indutor de resistência apresentaram reduções percentuais de 75,89\% (T1, T2 e T9), 79,04\% (T3), 26,42\% (T4, T6, T8), 47,09\% (T5), 0,24\% (T7), na severidade de doenças quando comparados com o tratamento controle (Tabela 5).

Quando comparado com tratamento controle, os tratamentos que receberam 3 aplicações de fungicida sem a combinação com o indutor de resistência (T1, T4, T7,) observou-se uma redução percentual de 27,54\% na severidade de doenças, entretanto, quando comparado os tratamentos T2, T5 e T8 onde aos 45 dias após plantio (DAP) foi realizada a aplicação do fungicida combinado com o indutor de resistência, observou-se uma redução na severidade de 40,46\% (Tabela 5).

Os melhores resultados foram observados nos tratamentos T3, T6, T9, onde foram feitas as combinações com indutor de resistência na $1^{a}$ e $2^{a}$ aplicação de fungicida, promovendo uma redução de $46,85 \%$ na severidade de doenças, tais resultados mostram a capacidade dos indutores em estimular a rota e os mecanismos de defesa nas plantas, o qual pode ser em uma região específica ou de forma sistêmica, promovendo um efeito sinérgico com os fungicidas.

As variáveis massa de 100 grãos (M100G) e a produtividade de grãos (PG) foram significativas em função dos tratamentos (Tabela 6).

Tabela 6. Resumo da análise de variância para as variáveis massa de 100 grãos (M100G) e produtividade de grãos (PG), em função dos tratamentos, safra 2018-19, Rio Verde - GO.

\begin{tabular}{cccc}
\hline \multirow{2}{*}{ FV } & GL & \multicolumn{2}{c}{ Quadrados médios (QM) } \\
\cline { 3 - 4 } & 9 & $0,5515^{* * *}$ & PG \\
\hline Tratamentos & 3 & $0,2879^{*}$ & $487734,7925^{* *}$ \\
Blocos & 27 & 0,0795 & $31004,2014^{\text {ns }}$ \\
Resíduos & & 1,55 & 143983,0728 \\
CV $(\%)$ & & 9,57 \\
\hline
\end{tabular}

${ }^{\text {ns }}$ não significativo e *; ** significativo respectivamente a 5 e $1 \%$ de probabilidade segundo teste F. GL - Grau de Liberdade e CV Coeficiente de Variação. Fonte: Autores.

O T3 apresentou a maior M100G, e quando comparada ao Controle promoveu um aumento de 6,74\%, entretanto o T3 não apresenta diferença dos tratamentos T1; T4; T6 e T8 que quando comparados ao Controle promoveram um aumento médio de 4,24\% na M100G. Os tratamentos T2; T5; T7 e T9 não diferem do Controle apesar de terem promovido um aumento médio de $1,86 \%$ (Tabela 7). 
Nos tratamentos sem o uso dos indutores: T1, T4 e T7, não diferiram significativamente do tratamento controle. Os tratamentos em que houve uma ou duas aplicações de fungicidas + indutor de resistência (T2, T3, T5, T6, T8, T9), apresentaram valores significativos em relação ao controle, com ganhos médios respectivos de 16,75\% (803,68 kg ha-1); $\left.31,52 \%\left(1016,15 \mathrm{~kg} \mathrm{ha}^{-1}\right) ; 29,10 \%\left(937,95 \mathrm{~kg} \mathrm{ha}^{-1}\right) ; 29,69 \%\left(956,91 \mathrm{~kg} \mathrm{ha}^{-1}\right) ; 25,08 \%(808,42 \mathrm{~kg} \mathrm{ha})^{-1}\right) ; 39,00 \%(1257,01 \mathrm{~kg}$ $\mathrm{ha}^{-1}$ ) (Tabela 4). O uso do indutor de resistência promoveu um incremento significativo de produtividade quando comparados aos resultados obtidos com o uso de fungicidas de forma isolada (Tabela 7).

Tabela 7. Teste de média para as variáveis massa de 100 grãos (M100G) e produtividade de grãos (PG), em função dos tratamentos, safra 2018-19, Rio Verde - GO.

\begin{tabular}{ccc}
\hline \multirow{2}{*}{ Tratamentos } & \multicolumn{1}{c}{ Médias } \\
\cline { 2 - 3 } 1 & M100G & PG ha \\
2 & $\mathrm{~g}$ & $3762,86 \mathrm{~b}$ \\
3 & $18,27 \mathrm{ab}$ & $4026,68 \mathrm{a}$ \\
4 & $17,68 \mathrm{bc}$ & $4089,86 \mathrm{a}$ \\
5 & $18,38 \mathrm{a}$ & $3742,33 \mathrm{~b}$ \\
6 & $17,79 \mathrm{abc}$ & $4160,95 \mathrm{a}$ \\
7 & $17,69 \mathrm{bc}$ & $4179,91 \mathrm{a}$ \\
8 & $17,88 \mathrm{abc}$ & $3812,63 \mathrm{~b}$ \\
9 & $17,38 \mathrm{c}$ & $4031,41 \mathrm{a}$ \\
Controle & $17,87 \mathrm{abc}$ & $4480,01 \mathrm{a}$ \\
\end{tabular}

Médias seguidas da mesma letra nas colunas não diferem entre si segundo teste Tukey a 1\% de probabilidade. Fonte: Autores.

Os resultados mostram que os mecanismos de defesa foram eficientemente estimulados, corroborando com os resultados observados por Silva et al. (2013), observaram resultados condizentes aos do presente estudo, e ainda afirmam existir uma relação entre a concentração dos indutores de resistência no tecido vegetal e a severidade dos fitopatógenos nas plantas (Spolti et al., 2015), principalmente na supressão da severidade de doenças causadas por fungos.

Produtos à base fosfito de cobre em combinação com os fungicidas apresentam um efeito sinérgico, refletindo no efeito supressivo sobre a severidade de doenças e na produtividade. O dinamismo entre os fungicidas e o fosfito aumenta a resposta imunológica da planta ao ataque de patógenos, induzindo a síntese sistêmica de fitoalexinas (Monteiro et al., 2014). O uso do indutor de resistência, em combinação com fungicidas, pode aumentar a eficiência de controle de doenças, além de reduzir a seleção de fitopatógenos resistentes. O uso de fosfitos em mistura com fungicidas incrementa na atividade residual de fertilizantes (Rosenberger \& Cox, 2009; Silva Cavalcante et al., 2020). Segundo Araújo et al. (2010) em teste feito in vitro os fosfitos possuem ação direta sobre a fisiologia dos patógenos.

\section{Conclusão}

O indutor de resistência em combinação com fungicidas reduziram a severidade e a incidência de doenças com uma ou duas aplicações.

Os tratamentos mais eficientes na redução da severidade de doenças foram aqueles que tiveram uma ou duas aplicações do Fosfito de $\mathrm{Cu}$.

$\mathrm{O}$ fosfito de $\mathrm{Cu}$ em associação com os diferentes fungicidas incrementou significativamente a produtividade de grãos de soja. 


\section{Agradecimentos}

Os autores agradecem à Fundação de Amparo à Pesquisa do Brasil (Conselho Nacional de Desenvolvimento Científico e Tecnológico - CNPq), à Coordenação de Aperfeiçoamento de Pessoal de Nível Superior (CAPES); a Fundação de Amparo à Pesquisa do Estado de Goiás (FAPEG); Ministério da Ciência, Tecnologia, Inovações e Comunicações (MCTIC Brasil); a Financiadora de Estudos e Projetos (Finep); Centro de Excelência em Agro Exponencial (CEAGRE); ao Instituto Federal de Educação, Ciência e Tecnologia Goiano (IF Goiano) - Campus Rio Verde; a Universidade de Rio Verde - UniRV; AGIRTEC - Soluções de Precisão; pelo apoio financeiro e estrutural para a realização deste estudo.

\section{Referências}

Araújo, L., Valdebenito-Sanhueza, R. M., \& Stadnik, M. J. (2010). Avaliação de formulações de fosfito de potássio sobre Colletotrichum gloeosporioides in vitro e no controle pós-infeccional da mancha foliar de Glomerella em macieira. Tropical Plant Pathology, 35, $054-059$.

Bertoldo, E., \& Mazaro, S. M. (2018). ß-1, 3 Glucanases: uma revisão sob a ótica da defesa vegetal. Scientia Agraria Paranaensis, 17(1), 1-13.

Carvalho, N. L. (2012). Resistência genética induzida em plantas cultivadas. Revista Eletrônica em Gestão, Educação e Tecnologia Ambiental, 7(7), 13791390 .

Silva Cavalcante, W. S., da Silva, N. F., Teixeira, M. B., Cabral Filho, F. R., Nascimento, P. E. R., \& Corrêa, F. R. (2020). Eficiência dos bioestimulantes no manejo do déficit hídrico na cultura da soja. IRRIGA, 25(4), 754-763. https://doi.org/10.15809/irriga.2020v25n4p754-763.

Companhia nacional de abastecimento - conab. Acompanhamento da safra brasileira de grãos, v7 - Safra 2019/20, n. 6 - Sexo levantamento, março de 2020. Brasília, 2020.

Ferreira, D. F. (2011). Sisvar: a computer statistical analysis system. Ciência e agrotecnologia, 35, 1039-1042.

Fonseca, E. M. S., \& Araújo, R. C. (2015). Fitossanidade, princípios básicos e metodos de controle de doenças e pragas. Editora Ér. org. RC Fonseca, EMS, Araújo. São Paulo, SP: Editora Érica-Saraíva.

Guest, D., \& Grant, B. (1991). The complex action of phosphonates as antifungal agents. Biological Reviews, 66(2), $159-187$.

Ioris Junior, M. A. (2019). Proteção de plantas de soja com tratamentos fungicidas associados à indução de resistência: efeitos no rendimento da cultura e qualidade de grãos. Trabalho de Conclusão de Curso. Universidade Federal de Mato Grosso - UFMT.

Juhász, A. C. P., de Pádua, G. P., Wruck, D. S. M., Favoreto, L., \& Ribeiro, N. R. (2013). Desafios fitossanitários para a produção de soja. Embrapa Agrossilvipastoril-Artigo em periódico indexado (ALICE).

Lovatt, C. J., \& Mikkelsen, R. L. (2006). Phosphite fertilizers: What are they? Can you use them? What can they do Better crops, 90(4), 11-13.

Martins, M. C., Guerzoni, R. A., Câmara, G. M. D. S., Mattiazzi, P., Lourenço, S. A., \& Amorim, L. (2004). Escala diagramática para a quantificação do complexo de doenças foliares de final de ciclo em soja. Fitopatologia Brasileira, 29, 179-184.

Meneghetti, R. C., Balardin, R. S., Corte, G. D., Favera, D. D., \& Debona, D. (2010). Avaliação da ativação de defesa em soja contra Phakopsora pachyrhizi em condições controladas. Ciência e Agrotecnologia, 34, 823-829.

Monteiro, A. C. A. (2014). Indutores de resistência no manejo da ferrugem e cercosporiose do cafeeiro (Coffea arabica): análises bioquímicas e moleculares. Tese de Doutorado. Tese (Doutorado em Agronomia - Fitopatologia) - Universidade Federal de Lavras, Lavras, 2014.132.

Ribeiro, F. C.; Colombo, G. A.; Carvalho, E. V.; Pelúzio, J. M.; Erasmo, E. A. L. (2017). Controle químico de mancha-alvo da soja (Corynespora cassiicola) no cerrado tocantinense -Brasil. Gurupi, Universidade Federal do Tocantins, 26-36.

Ribeiro, L. A. E., Júnior, D. P. M., de Sá, D. D. D., de Araújo, F. P., \& de Souza, J. E. B. (2019). Viabilidade Econômica Do Uso De Fungicidas No Controle Da Ferrugem Asiática Da Soja. Ipê Agronomic Journal, 3(2), 35-43.

Rosenberger, D. A., Cox, K. D., \& Highland, N. Y. (2009). Using phosphite fungicides to control apple diseases. New York Fruit Quarterly, Geneva, 17, 9-13.

Santos, H. G., Jacomine, P. K. T., Dos Anjos, L. H. C., De Oliveira, V. A., Lumbreras, J. F., Coelho, M. R., \& CunhA, T. J. F. (2018). Sistema brasileiro de classificação de solos. Brasília, DF: Embrapa, 2018.

Silva, O. C., Santos, H. A., Deschamps, C., Dalla Pria, M., \& May De Mio, L. L. (2013). Fontes de fosfito e acibenzolar-S-metílico associados a fungicidas para o controle de doenças foliares na cultura da soja. Tropical Plant Pathology, 38, 72-77.

Spolti, P., Valdebenito-Sanhueza, R. M., Campos, Â. D., \& Del Ponte, E. M. (2015). Modo de ação de fosfitos de potássio no controle da podridão olho de boi em maçã. Summa Phytopathologica, 41, 42-48.

Taiz, L.; \& Zeiger, E. (2017). Fisiologia vegetal. (6a ed.), Artemed. 